\title{
Control system modeling for superconducting accelerator
}

\author{
Tomasz Czarski, Krzysztof Pozniak, Ryszard Romaniuk, \\ Institute of Electronic Systems, Warsaw University of Technology \\ Stefan Simrock \\ DESY, Hamburg
}

\begin{abstract}
A digital control of superconducting cavities for a linear accelerator is presented. The LLRF - Low Level Radio Frequency system for FLASH project in DESY is introduced. FPGA based controller supported by MATLAB system was developed to investigate the novel firmware implementation. Algebraic model in complex domain is proposed for the system analyzing. Calibration procedure of a signal path is considered for a multi-channel control. Identification of the system parameters is carried out by the least squares method application. Control tables: Feed-Forward and SetPoint are determined for the required cavity performance, according to the recognized process. Feedback loop is tuned by fitting a complex gain of a corrector unit. Adaptive control algorithm is applied for feed-forward and feedback modes. Experimental results are presented for a cavity representative operation.
\end{abstract}

Keywords: superconducting cavity control, system identification, LLRF control.

\section{INTRODUCTION}

LLRF control system is under development in order to improve regulation of accelerating fields in the resonators [1] [4] (fig. 1). Control section, powered by one klystron, may consist of many cavities. Fast amplitude and phase control of the cavity field is accomplished by modulation of a signal driving the klystron through a vector modulator. Cavities are driven with $1.3 \mathrm{~ms}$ pulses to an average accelerating gradient of $25 \mathrm{MV} / \mathrm{m}$. The cavity RF signal is down-converted to an intermediate frequency of $250 \mathrm{KHz}$, while preserving the amplitude and phase information. ADC and DAC converters link the analog and digital parts of the system with a sampling interval of $1 \mu$ s. Digital signal processing is executed in the FPGA system to obtain field vector detection, calibration and filtering. Control feedback system regulates the vector sum of the pulsed accelerating fields in multiple cavities. The FPGA based controller stabilizes the detected real (in-phase) and imaginary (quadrature) components of the incident wave according to a given set point. Adaptive feed-forward is applied to improve the compensation of repetitive perturbations induced by the beam loading and by dynamic Lorentz force detuning. Control block applies the value of the cavity parameters, estimated in the identification system, and generates the required data for the controller. A system model was developed for investigating the optimal control method of the cavity. The control system was experimentally introduced in the first cryo-module with 8 cavities - ACC1 of the VU-FEL TTF (FLASH) in DESY.

\section{CAVITY CONTROL SYSTEM MODELING}

\subsection{Single channel modeling and calibration procedure}

A discrete-time model in complex domain is introduced to analyze the LLRF digital control system (fig. 2) [2]. A signal is modeled by a complex envelope called "phasor". It is represented by real (in-phase) and imaginary (quadrature) components or alternatively by amplitude and phase, related to the reference frequency of $1.3 \mathrm{GHz}$ of the carrier signal. Complex modules of the system form a phasor according to their dynamic or static and linear or nonlinear characteristics. The considerations are for a single cavity without a beam.

Electrical model of the cavity is assumed as the only dynamic part. The discrete model is based on a difference equation of first order for the output phasor v', driven with the input phasor $\mathbf{u}$. The recursive equation of the cavity model, with sampling interval $\mathrm{T}$, is expressed by the complex form, for a step $\mathrm{k}$ :

$$
\mathbf{v}^{\prime}{ }_{\mathrm{k}+1}=\mathbf{E}_{\mathrm{k}} \cdot \mathbf{v}^{\prime}{ }_{\mathrm{k}}+\mathbf{A} \cdot \mathbf{u}_{\mathrm{k}},
$$

where the linear input factor is $\mathbf{A}$, and the non linear system factor is $\mathbf{E}_{\mathrm{k}}=\left(1-\mathrm{T} \omega_{1 / 2}\right)+\mathrm{T} \Delta \omega_{\mathrm{k}} \cdot \boldsymbol{i}$, with cavity parameters: constant half-bandwidth $=\omega_{1 / 2}$ and detuning $=\Delta \omega_{\mathrm{k}}$, time varying during a pulse. 
The internal cavity output $\mathbf{v}_{\mathrm{k}}$ is not available directly for the control purpose. Cavity environment is divided for two static parts represented by the linear output unit $\mathbf{B}$ and the non linear klystron unit with time varying factor $\mathbf{D}_{\mathrm{k}}$. $\mathbf{A}$ calibration of the linear path, characterized by factor $\mathbf{A}$ and $\mathbf{B}$, is essential for a cavity control, and is required in a multichannel case. Scaling and phasing of a single channel is accomplished by means of a calibrator unit $\mathbf{C}$ implemented inside the FPGA controller area.

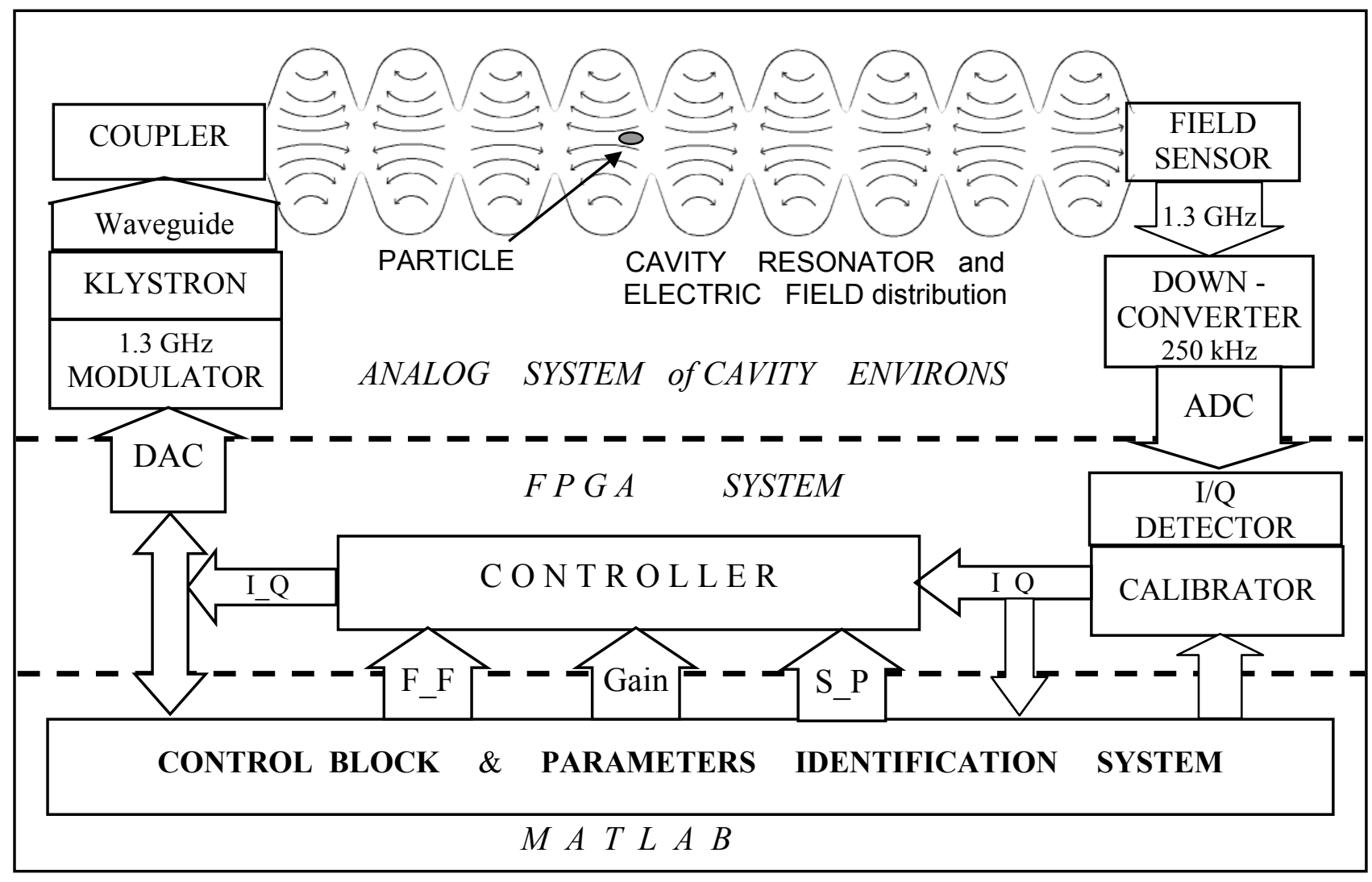

Fig 1. Functional block diagram of LLRF control system for one cavity

The resulting, external cavity model driven with a klystron phasor $\mathbf{u}_{\mathrm{k}}$, seen by actual controller for a substituted cavity phasor $\mathbf{v}_{\mathrm{k}}=\mathbf{B} \cdot \mathbf{C} \cdot \mathbf{v}_{\mathrm{k}}$, is expressed for step $\mathrm{k}$ :

$$
\mathbf{v}_{\mathrm{k}+1}=\mathbf{E}_{\mathrm{k}} \cdot \mathbf{v}_{\mathrm{k}}+\mathbf{F} \cdot \mathbf{u}_{\mathrm{k}}
$$

where the substituted factor $\mathbf{F}=\mathbf{A} \cdot \mathbf{B} \cdot \mathbf{C}$ represents a static and linear characteristic of a single channel.

Calibration procedure applies the cavity field gradient $\left|\mathbf{v}^{\prime}{ }_{\mathrm{k}}\right|$ to fulfill the scaling condition: $\left|\mathbf{v}_{\mathrm{k}}\right|=\left|\mathbf{v}_{\mathrm{k}}{ }^{\prime}\right|$. A phase calibration fulfills the condition: $\arg (\mathbf{F})=0$, when $\arg \left(\mathbf{v}_{\mathrm{k}}\right) \rightarrow \arg \left(\mathbf{u}_{\mathrm{k}}\right)$ for $\mathrm{k} \rightarrow 0$. Careful estimation of cavity phasor $\mathbf{v}_{\mathrm{k}}$ and klystron phasor $\mathbf{u}_{\mathrm{k}}$ is required for a small gradient in a beginning of pulse. The phase calibration can be verified, if $\arg \left(\mathbf{v}_{\mathrm{k}}\right)=\arg \left(\mathbf{u}_{\mathrm{k}}\right)$ in a resonance condition for two cases:

1. in a flattop steady state: $\mathbf{v}_{\mathrm{k}}=$ const. and $\Delta \omega_{\mathrm{k}}=0$

2. in a filling range for modulated phase: $\varphi_{\mathrm{k}}=\varphi_{\mathrm{k}-1}+\mathrm{T} \cdot \Delta \omega_{\mathrm{k}}$, where $\varphi_{\mathrm{k}}=\arg \left(\mathbf{u}_{\mathrm{k}}\right)$.

Actually, the calibration procedure is coupled with parameters identification (chapter 3) and control algorithm (chapter 4 ), which are performed adaptively. 


\subsection{Multi channel system modeling}

In a practical application of a linear accelerator one klystron drives many cavities. Therefore, parallel multi-channel system, driven with a common klystron phasor $\mathbf{u}_{\mathrm{k}}$, for vector sum control is considered. Each single cavity $i$-th channel with a cavity phasor $\mathbf{v}_{\mathrm{k}}^{\mathrm{i}}$, is modeled according to the equation (2), for step k:

$$
\mathbf{v}_{\mathrm{k}+1}^{\mathrm{i}}=\mathbf{E}_{\mathrm{k}}^{\mathrm{i}} \cdot \mathbf{v}_{\mathrm{k}}^{\mathrm{i}}+\mathrm{F}^{\mathrm{i}} \cdot \mathbf{u}_{\mathrm{k}},
$$

where the calibrated factor $\mathrm{F}^{\mathrm{i}}$ has a scalar value and system factor is $\mathbf{E}_{\mathrm{k}}^{\mathrm{i}}=\left(1-\mathrm{T} \omega_{1 / 2}^{\mathrm{i}}\right)+\mathrm{T} \Delta \omega_{\mathrm{k}}^{\mathrm{i}} \cdot \boldsymbol{i}$ for $i$-th cavity.

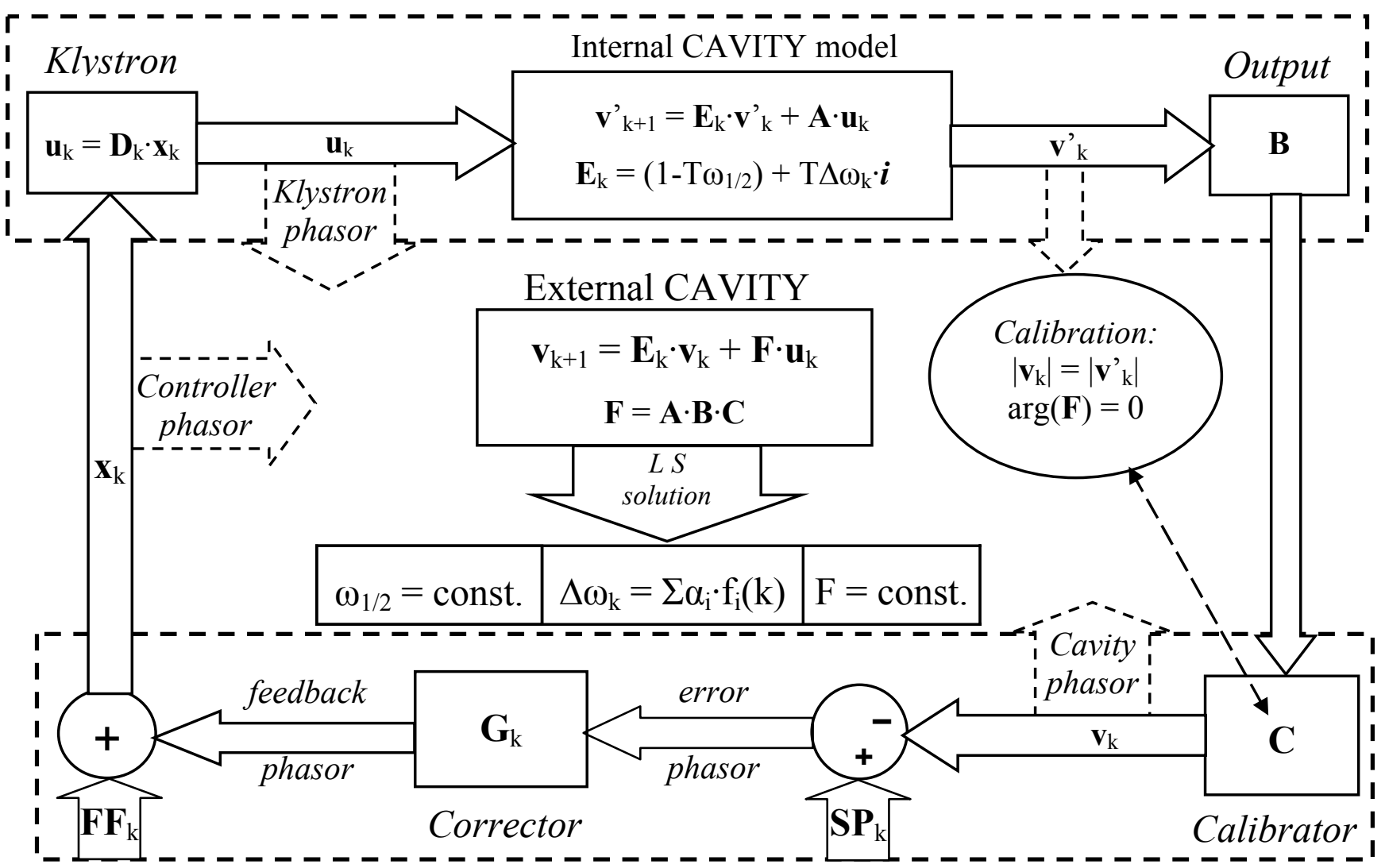

Fig 2. Algebraic model of LLRF control system

Summarizing equation (3) for all channels and introducing new variables (but familiar notation) yields, for step k:

$$
\mathbf{v}_{\mathrm{k}+1}=\mathbf{E}_{\mathrm{k}} \cdot \mathbf{v}_{\mathrm{k}}+\mathrm{F} \cdot \mathbf{u}_{\mathrm{k}}
$$

where resultant cavity phasor is $\mathbf{v}_{\mathrm{k}}=\sum_{\mathrm{i}}\left(\mathbf{v}_{\mathrm{k}}^{\mathrm{i}}\right)$, resultant input factor is $\mathrm{F}=\sum_{\mathrm{i}}\left(\mathrm{F}^{\mathrm{i}}\right)$, and equation: $\mathbf{E}_{\mathrm{k}} \cdot \mathbf{v}_{\mathrm{k}}=\sum_{\mathrm{i}}\left(\mathbf{E}_{\mathrm{k}}^{\mathrm{i}} \cdot \mathbf{v}_{\mathrm{k}}^{\mathrm{i}}\right)$ defines a weighted average system factor $\mathbf{E}_{\mathrm{k}}=\left(1-\mathrm{T} \omega_{1 / 2}\right)+\mathrm{T} \Delta \omega_{\mathrm{k}} \cdot \boldsymbol{i}$, with resultant half-bandwidth $=\omega_{1 / 2}$ and resultant detuning $=$ $\Delta \omega_{\mathrm{k}}$. Consequently, the multi-cavity model introduced by equation (4) has the same structure like calibrated single channel model (2), but with new parameters in the case of diverse operational condition for cavities.

Including the klystron unit (fig. 2) to the cavity system (4), the ultimate model, driven with the controller phasor $\mathbf{x}_{\mathrm{k}}$, is given by:

$$
\mathbf{v}_{\mathrm{k}+1}=\mathbf{E}_{\mathrm{k}} \cdot \mathbf{v}_{\mathrm{k}}+\mathbf{H}_{\mathrm{k}} \cdot \mathbf{x}_{\mathrm{k}}
$$

where total factor $\mathbf{H}_{\mathrm{k}}=\mathrm{F} \cdot \mathbf{D}_{\mathrm{k}}$. 


\subsection{Controller modeling}

FPGA based controller executes procedure of feed-forward driving supported by feedback according to prearranged control tables: $\mathbf{F F}_{\mathrm{k}}, \mathbf{S P}_{\mathrm{k}}, \mathbf{G}_{\mathrm{k}}$ (fig. 2). A cavity phasor $\mathbf{v}_{\mathrm{k}}$ is compared to the reference phasor $\mathbf{S P}_{\mathrm{k}}$ (set point) creating an error phasor. An error phasor is multiplied by a complex gain $\mathbf{G}_{\mathrm{k}}$ of the corrector unit, producing a feedback phasor. A superposition of a feedback phasor and a compensating phasor $\mathbf{F F}_{\mathrm{k}}$ (feed-forward) results in a controller phasor $\mathbf{x}_{\mathrm{k}}$. Consequently, the controller model is expressed for step k:

$$
\mathbf{x}_{\mathrm{k}+1}=\mathbf{F F}_{\mathrm{k}}+\mathbf{G}_{\mathrm{k}} \cdot\left(\mathbf{S P}_{\mathrm{k}}-\mathbf{v}_{\mathrm{k}}\right) \text {. }
$$

Control tables are determined for required cavity performance, according to the control algorithm described in chapter 4.

\section{PARAMETERS IDENTIFICATION OF CAVITY SYSTEM}

Control algorithm (chapter 4), based on the cavity system model (5), requires identification of the process parameters [3]. Therefore, two complex, time varying factors $\mathbf{E}_{\mathrm{k}}$ and $\mathbf{H}_{\mathrm{k}}$, should be recognized in the equation (5). The LS - least square method is proposed for parameters estimation in a noisy and non stationary condition. This method is efficient for uncorrelated variables and for the model structure fitting to the reality.

The first stage of the estimation procedure engages linear part of the model expressed by equation (4), where factor $\mathrm{F}$ has constant scalar value. Complex equation (4) is expanded to a scalar form, applying the real $\left(\mathrm{v}_{\mathrm{r}}, \mathrm{u}_{\mathrm{r}}\right)$ and imaginary $\left(\mathrm{v}_{\mathrm{i}}, \mathrm{u}_{\mathrm{i}}\right)$ components of the cavity and klystron phasor respectively, for step $\mathrm{k}$ and $\mathrm{k}+1$, as follows:

$$
\begin{aligned}
\left(v_{r}\right)_{k+1} & =\left(1-T \omega_{1 / 2}\right) \cdot\left(v_{r}\right)_{k}-T \Delta \omega_{k} \cdot\left(v_{i}\right)_{k}+F \cdot\left(u_{r}\right)_{k} \\
\left(v_{i}\right)_{k+1} & =\left(1-T \omega_{1 / 2}\right) \cdot\left(v_{i}\right)_{k}+T \Delta \omega_{k} \cdot\left(v_{r}\right)_{k}+F \cdot\left(u_{i}\right)_{k} .
\end{aligned}
$$

Three scalar unknowns include two stable parameters: half-bandwidth $\omega_{1 / 2}$ and factor $\mathrm{F}$, and step varying detuning $\Delta \omega_{\mathrm{k}}$. These parameters should be estimated for each k-th step of the process, described by two equations (7) and (8), involving measured input and output data. Time varying detuning $\Delta \omega_{\mathrm{k}}$ can be approximated for each k-th step by Lorder series of base functions $f_{j}$ with unknown, but constant coefficients $\alpha_{j}$, as follows:

$$
\mathrm{T} \Delta \omega_{\mathrm{k}}=\sum_{\mathrm{j}=1}^{\mathrm{L}} \alpha_{\mathrm{j}} \mathrm{f}_{\mathrm{j}}(\mathrm{k})=\mathbf{f}_{\mathrm{k}} \cdot \boldsymbol{\alpha},
$$

where $\boldsymbol{\alpha}$ - column vector of $\mathrm{L}$ coefficients, $\mathbf{f}_{\mathrm{k}}$ - row vector of $\mathrm{L}$ values of chosen base functions for k-th step.

Substituting equation (9) to (7) and (8), all unknowns are extracted to the vector form and matrix equation with MATLAB convention is introduced, as follows:

$$
\mathbf{v}_{\mathrm{k}+1}=\mathbf{w}_{\mathrm{k}} \cdot \mathbf{z}
$$

where $\mathbf{z}=\left[\left(1-T \omega_{1 / 2}\right) ; \boldsymbol{\alpha} ; F\right]$ is resultant column vector of unknown $L+2$ values,

$\mathbf{v}_{\mathrm{k}}=\left[\left(\mathrm{v}_{\mathrm{r}}\right)_{\mathrm{k}} ;\left(\mathrm{v}_{\mathrm{i}}\right)_{\mathrm{k}}\right], \quad \mathbf{w}_{\mathrm{k}}=\left[\mathbf{v}_{\mathrm{k}}, \mathbf{v}^{{ }_{\mathrm{k}}} \cdot \mathbf{f}_{\mathrm{k}}, \mathbf{u}_{\mathrm{k}}\right]$ for $\mathbf{v}_{\mathrm{k}}{ }_{\mathrm{k}}=\left[-\left(\mathrm{v}_{\mathrm{i}}\right)_{\mathrm{k}} ;\left(\mathrm{v}_{\mathrm{r}}\right)_{\mathrm{k}}\right]$ and $\mathbf{u}_{\mathrm{k}}=\left[\left(\mathrm{u}_{\mathrm{r}}\right)_{\mathrm{k}} ;\left(\mathrm{u}_{\mathrm{i}}\right)_{\mathrm{k}}\right]$.

In a practical application of LS method, equation (10) is considered for $\mathrm{N}$ steps of an approximation range, creating $2 \mathrm{~N}$ over-determined equations $(2 \mathrm{~N}>>\mathrm{L}+2)$, expressed by the matrix form, as follows:

$$
\mathbf{V}=\mathbf{W} \cdot \mathbf{z}
$$

where, $\quad \mathbf{V}$ - total output vector $(\operatorname{dim}=2 \mathrm{~N} \times 1), \quad \mathbf{W}-$ total matrix of model structure $(\operatorname{dim}=2 \mathrm{~N} x \mathrm{~L}+2)$.

Multiplying two sides of equation (11) by matrix transposition $\mathbf{W}^{\mathrm{T}}$, the solution for the vector $\mathbf{z}$ is given by:

$$
\mathbf{z}=\left(\mathbf{W}^{\mathrm{T}} * \mathbf{W}\right)^{-1} * \mathbf{W}^{\mathrm{T}} * \mathbf{V}
$$

It is the unique and optimal solution, according to the LS method for the measured data of vector $\mathbf{V}$ and structure matrix W. Algorithm for the identification of cavity parameters was implemented in Matlab system applying cubic B-spline set of functions for linear decomposition of step-varying detuning [3].

Second stage of the estimation procedure engages non linear part of the model described by the complex equation $\mathbf{u}_{\mathrm{k}}=$ $\mathbf{D}_{\mathrm{k}} \cdot \mathbf{x}_{\mathrm{k}}$ for the klystron unit (fig 2). Finally the factor $\mathbf{H}_{\mathrm{k}}$ from equation (5) is estimated, as follows: $\mathbf{H}_{\mathrm{k}}=\mathrm{F} \cdot \mathbf{u}_{\mathrm{k}} / \mathbf{x}_{\mathrm{k}}$. The prior estimation (filtering) for measured data - cavity, controller and klystron phasor, was performed for of-line identification of the parameters presented in this chapter. 


\section{CONTROL of CAVITY SYSTEM}

\subsection{Feed-forward and feedback driving}

The required cavity performance is: driving in resonance during filling and field stabilization for flattop range. A cavity is driven in feed-forward and feedback mode to fulfill desired operation condition. Combining equation (5) and (6), yield resulting equation for the cavity control system, as follows:

$$
\mathbf{v}_{\mathrm{k}+1}=\mathbf{E}_{\mathrm{k}} \cdot \mathbf{v}_{\mathrm{k}}+\mathbf{H}_{\mathrm{k}} \cdot\left[\mathbf{F F}_{\mathrm{k}-1}+\mathbf{G}_{\mathrm{k}-1} \cdot\left(\mathbf{S P}_{\mathrm{k}-1}-\mathbf{v}_{\mathrm{k}-1}\right)\right]
$$

Recognition of the cavity system factors: $\mathbf{E}_{\mathrm{k}}$ and $\mathbf{H}_{\mathrm{k}}$, allows determining the control tables: feed-forward - $\mathbf{F F}_{\mathrm{k}}$, set point - $\mathbf{S P}_{\mathrm{k}}$ and complex gain $-\mathbf{G}_{\mathrm{k}}$. The required cavity phasor $\mathbf{v}_{\mathrm{k}}$ can be achieved by ideal feed-forward compensation according to the recognized system model. However, feedback mode compensates a stochastic disturbances and a model discrepancy.

Let's consider equation (13) separately for two modes of operation, reduced as

$$
\mathbf{v}_{\mathrm{k}+1}=\mathbf{E}_{\mathrm{k}} \cdot \mathbf{v}_{\mathrm{k}}+\mathbf{U}_{\mathrm{k}}
$$

where $\mathbf{U}_{\mathrm{k}}=\mathbf{H}_{\mathrm{k}} \cdot \mathbf{F} \mathbf{F}_{\mathrm{k}-1}$ for feed-forward or $\mathbf{U}_{\mathrm{k}}=\mathbf{H}_{\mathrm{k}} \cdot \mathbf{G}_{\mathrm{k}-1} \cdot\left(\mathbf{S} \mathbf{P}_{\mathrm{k}-1}-\mathbf{v}_{\mathrm{k}-1}\right)$ for feedback.

Solution of equation (14) gives estimated formulas presented in tab. 1, for two modes of operation within two ranges. Complex gain $\mathbf{G}_{\mathrm{k}}$ is determined for the loop gain $\mathrm{L}$ assumed as constant scalar parameter for the steady state cavity phasor $\underline{\mathbf{v}}_{\mathrm{k}}=\mathrm{L} \cdot\left(\mathbf{S P}_{\mathrm{k}}-\underline{\mathbf{v}}_{\mathrm{k}}\right)$. The loop gain $\mathrm{L}$ value is limited due to the stability condition.

- $\quad$ Filling range

Equation (14) is driven with phasor $\mathbf{U}_{\mathrm{k}}$ with a constant amplitude and modulated phase $\varphi_{\mathrm{k}}$, so the RF signal tracks a step-varying resonance frequency of a cavity. In a response, the cavity phasor $\mathbf{v}_{\mathrm{k}}$ has the same phase and exponential raise of amplitude, according to the natural behavior in the resonance condition. Initial phase $\varphi_{1}$ and asymptotic amplitude $\mathrm{v}$ are determined to fulfill condition for a final filling phasor $\mathbf{v}_{\text {end }}$, which equals to the required flattop phasor V.

- Flattop range

Equation (14) is driven with phasor $\mathbf{U}_{\mathrm{k}}$ compensating step varying factor $\mathbf{E}_{\mathrm{k}}$, so the cavity phasor $\mathbf{v}_{\mathrm{k}}$ is stabilized.

\begin{tabular}{|c|c|c|c|}
\hline Range & Fable & SET-POINT & GAIN \\
\hline Filling & $\mathbf{F F}_{\mathrm{k}}=\mathrm{T} \omega_{1 / 2} \cdot \mathrm{v} \cdot \exp \left(\boldsymbol{i} \varphi_{\mathrm{k}+1}\right) / \mathbf{H}_{\mathrm{k}+1}$ & $\mathbf{S P}_{\mathrm{k}}=\mathrm{v} \cdot\left[1-\exp \left(-\mathrm{k} \cdot \mathrm{T} \omega_{1 / 2}\right)\right] \cdot \exp \left(\boldsymbol{i} \varphi_{\mathrm{k}}\right)$ & $\mathbf{G}_{\mathrm{k}}=\mathrm{L} \cdot \mathrm{T} \omega_{1 / 2} / \mathbf{H}_{\mathrm{k}+1}$ \\
\hline Flattop & $\boldsymbol{\varphi}_{\mathrm{k}+1}=\varphi_{\mathrm{k}}+\mathrm{T} \Delta \omega_{\mathrm{k}}=\mathbf{V} \cdot \mathrm{T}\left(\omega_{1 / 2}-\Delta \omega_{\mathrm{k}+1} \cdot \boldsymbol{i}\right) / \mathbf{H}_{\mathrm{k}+1}$ & $\mathbf{S P}_{\mathrm{k}}=\mathbf{V}=\mathrm{V} \cdot \exp (\boldsymbol{i} \Phi)$ & $\mathrm{L}=$ const. \\
\hline
\end{tabular}

\subsection{Adaptive control process}

Superconducting cavity control is carried out according to the scheme of fig. 3. Control data, generated by Matlab system, is loaded to the internal FPGA memory and actuates the controller. The input and output data of the cavity is acquired to another area of the memory during a pulse operation. Subsequently, acquired data are conveyed to Matlab system for the parameters identification processing between pulses. Estimated cavity parameters are considered as actual values for the required cavity performance and are applied to create the control tables for a next pulse. But new control tables modify the trajectory of the nonlinear process and again new parameters are estimated. This iterative processing quickly converges to the desired state of the cavity, assuming repeatable conditions for successive pulses. Stochastic fluctuations of the required trajectory are reduced by averaging processing (filtering) for successive pulses.

The experimental results of adaptive control in the real operation condition are presented in fig. 4 for feed-forward driving. The cavity is activated with pulse of $1.3 \mathrm{~ms}$ duration and repetition of $10 \mathrm{~Hz}$. During the first stage of the operation $(\sim 0.5 \mathrm{~ms})$ the cavity is filling with constant forward power resulting in exponential increase of EM field in the resonance condition. When cavity phasor has reached the required final value, the steady state is forced during flattop range $(\sim 0.8 \mathrm{~ms})$. The initial predetuning attempts to compensate the Lorentz force detuning and balances the required 
power consumption during pulse operation of the cavity. Switching off the klystron power yields an exponential decay of the cavity field.

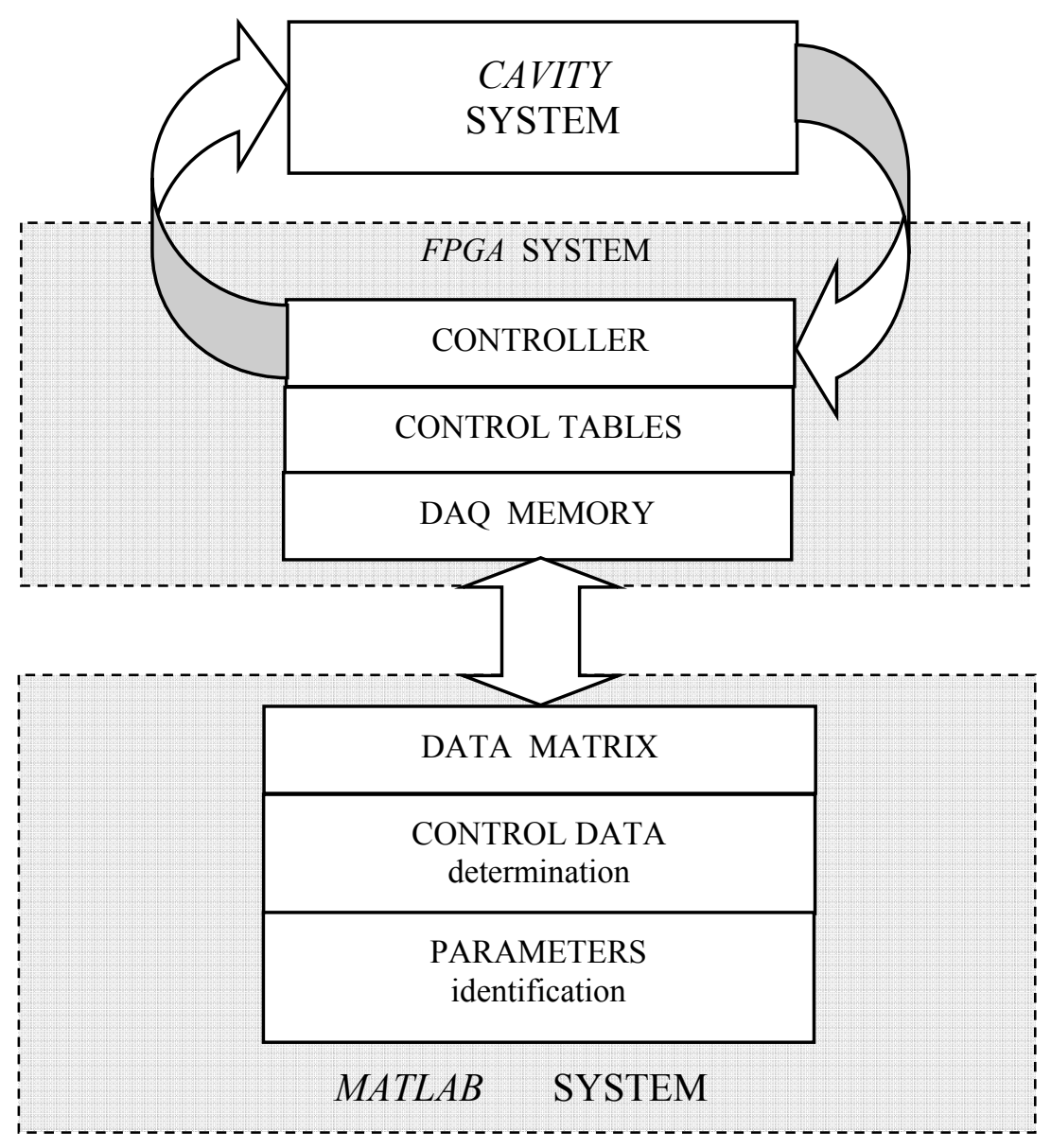

Fig 3. Adaptive control process for cavity system driving

\section{CONCLUSIONS}

The cavity control system for superconducting linear accelerator project is preliminary introduced in this paper. Digital control of the superconductive cavity has been performed by applying FPGA technology system in DESY. These experiments focused attention on the general recognition of the cavity features and projected control methods. Calibration and correction of the signal path is considered for the efficient driving of a cavity. Identification of the resonator parameters has been proven to be a successful approach in achieving required performance, i.e. driving on resonance during filling and field stabilization during flattop time while requiring reasonable levels of power consumption. Feed-forward and feedback modes were successfully applied in operating the cavity. Representative results of the experiments are presented for the typical operational condition. Preliminary application tests of FPGA controller have been carried out using the superconducting cavities in ACC1 module of the VUV-FEL (FLASH) setup.

\section{ACKNOWLEDGMENT}

We acknowledge the support of the European Community Research Infrastructure Activity under the FP6 "Structuring the European Research Area" program (CARE, contract number RII3-CT-2003-506395). 


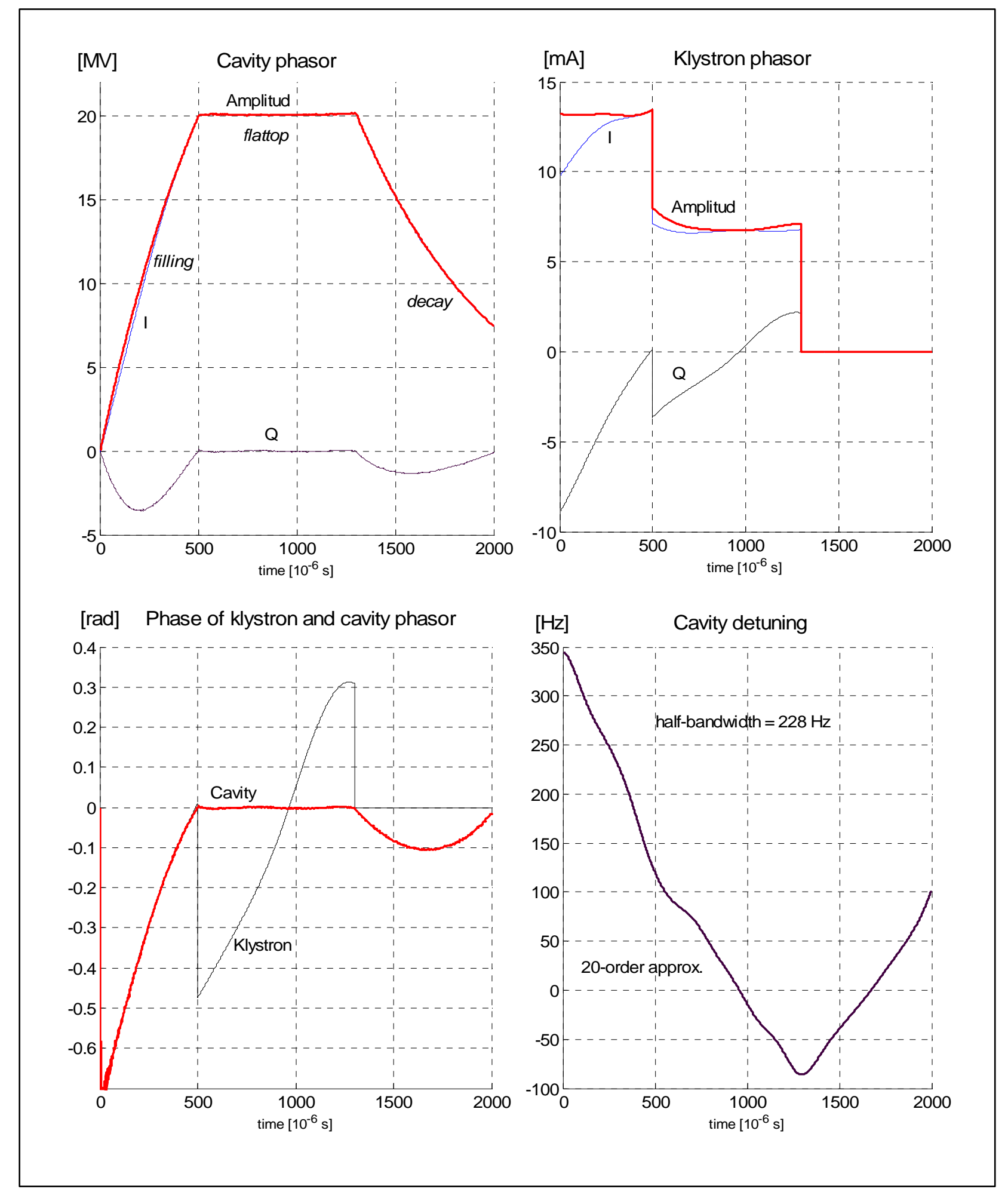

Figure 4. Feed-forward driving for single cavity of ACC1 module: cavity and klystron phasor readout, cavity detuning estimation for $20 \mathrm{MV}$ flattop level 


\section{REFERENCES}

1. T. Schilcher, "Vector Sum Control of Pulsed Accelerating Fields in Lorentz Force Detuned Superconducting Cavities", Ph. D. thesis, Hamburg, 1998.

2. T. Czarski, R.S. Romaniuk, K.T. Pozniak, and S. Simrock: "TESLA cavity modeling and digital implementation in FPGA technology for control system development", Nuclear Instruments and Methods in Physics Research Section A: Accelerators, Spectrometers, Detectors and Associated Equipment -Volume 556, Issue 2, 15 January 2006, Pages 565-576.

3. T. Czarski, R.S. Romaniuk, K.T. Pozniak, S. Simrock: Cavity parameters identification for TESLA control system development, Nuclear Instruments and Methods in Physics Research Section A: Accelerators, Spectrometers, Detectors and Associated Equipment -Volume 548, Issue 3, 21 August 2005, Pages 283-618.

4. http://www.flash.desy.de [Deutsches Elektronen-Synchrotron DESY] 